\title{
Quantized conductance of Si atomic wires
}

\author{
José-Luis Mozos, C. C. Wan, and Gianni Taraschi \\ Centre for the Physics of Materials and Department of Physics, McGill University, Montreal, Quebec, Canada H3A 2T8 \\ Jian Wang \\ Department of Physics, The University of Hong Kong, Pokfulam Road, Hong Kong, China \\ Hong Guo \\ Centre for the Physics of Materials and Department of Physics, McGill University, Montreal, Quebec, Canada H3A 2T8
}

(Received 16 April 1997)

\begin{abstract}
We have performed first-principles pesudopotential calculations of the quantum transport properties of a chain of $\mathrm{Si}$ atoms connected to the outside through long leads. By solving a three-dimensional quantum scattering problem we have computed the conductance for several atomic wires with up to eight $\mathrm{Si}$ atoms. The $\mathrm{Si}$ atomic wires are found to be metallic and we observed quantized conductance in units of $2 e^{2} / h$. A conductance dip is found to develop near the onset of the second quantized plateau as the number of atoms increases, and this can be explained by the existence of a gap in the density of states when the atomic chain is infinitely long. [S0163-1829(97)52032-7]
\end{abstract}

In the last decade extensive research effort has been devoted to the development of Si based optoelectronic technology. A particularly exciting result is the production of porous $\mathrm{Si}$ microstructures which have visible light photoluminescence. ${ }^{1}$ More recently, Si based atomic wires with sizes as small as several nanometers across, have been fabricated by several laboratories. ${ }^{2-5}$ Quantized conductance in units of $2 e^{2} / h$ is expected at elevated temperatures including room temperature, for atomic systems due to the large energy level separation of the transport channels. For the usual compound semiconductor submicron structures operating with the two-dimensional electron gas confined at a heterostructure, conductance quantization can only be observed at very low temperatures. Clearly these new research directions of Si based technology will certainly lead to new discoveries of nano-science and possibly to novel quantum electronic applications. In this regard, several very interesting device application directions have been discussed in the literature. $^{6}$

Using a scanning tunneling microscope (STM) one can measure the quantum conductance of a tip near a substrate, and quantized conductance has indeed been observed at room temperature ${ }^{7-11}$ for a number of tip-substrate systems. For Si based freestanding atomic wires, Ref. 2 reported observations of conductance quantization up to a temperature of $210 \mathrm{~K}$, and Coulomb blockade effect up to $100 \mathrm{~K}$. So far, theoretical analysis has largely focused on the important problem of quantum conduction through the tip-substrate arrangement. ${ }^{10,12-14}$ In particular, first-principles pseudopotential calculations for Si based nanowires have been carried out for optical properties to explain certain observed features of the porous silicon. ${ }^{15,16}$ Lang $^{14}$ has advanced a firstprinciples quantum scattering theory to study quantum conduction through atomic scale tip-substrate systems and the theoretical predictions for the case of a few Xe atoms sandwiched in between two substrates are in quantitative agreement with STM measurements. ${ }^{11}$ The authors have made $a b$ initio calculations of quantum transport through $\mathrm{Al}$ based freestanding atomic wires and predicted $\mathrm{dc}$ as well as ac conductances for these wires. ${ }^{17}$ Furthermore, Mehrez et al. have explained observed features of electric conduction through an atomic junction based on the behavior of density of states, ${ }^{18}$ and Watanabe et al. investigated the electronic band structure of the dangling-bond $\mathrm{Si}$ wires. ${ }^{19}$

Clearly, due to the importance of the problem, there is a need to further investigate quantum conduction through various atomic wire systems. It is also important and useful to directly compute quantum conductance from first principles by solving the quantum scattering matrix. Results from this kind of approach are especially strong in their predictive power as shown by the impressive results of Ref. 11 . The purpose of this work is to report our investigation on $\mathrm{Si}$ based freestanding wires using an ab initio method. Si wires are interesting and important, as they are the base semiconductor material of the electronics industry and $\mathrm{Si}$ atomic wires have been fabricated, as mentioned above. In particular, we shall consider the atomic wire model shown in the inset of Fig. 2, where a chain of several $\mathrm{Si}$ atoms is connected to the outside by three-dimensional (3D) leads. Electrons come in from the left lead, scatter off the Si atoms, and transmit to the right lead or reflect back. By solving the quantum scattering process using first principles, we compute the conductance for the whole system which can be viewed as a long 3D wire with an atomic chain in the middle. Our results will then be compared with those of metallic Al wires. $^{17}$

To solve the quantum conduction problem including the atomic degrees of freedom, we have developed a two-step numerical procedure. ${ }^{20,17}$ In the first step, we solve the ground-state properties of the $\mathrm{Si}$ chain and the left and right leads by minimizing the Kohn-Sham total-energy functional, where the valence electrons are treated explicitly and the core is introduced through a pseudopotential. We have fixed the ionic positions in our ground-state calculation. The well- 
known Kohn-Sham energy functional in standard density functional theory is the subject of many review articles, e.g., in Ref. 21, and to save space we refer interested readers to them. The equilibrium analysis produces the self-consistent Kohn-Sham electronic wave functions $\psi_{i}(\mathbf{r})$ with a selfconsistent effective potential $V_{e f f}(\mathbf{r}) \equiv \delta U / \delta \rho(\mathbf{r})$ which is seen by all the electrons. Here $U[\rho]$ is the total selfconsistent potential energy while $\rho$ the electron density obtained from $\left\{\psi_{i}\right\} .{ }^{21,22}$ We have used the parameterization of Ref. 23 for the exchange-correlation term, and a local pseudopotential $^{24}$ for the core. The second step of our calculation involves the evaluation of the scattering matrix of an electron traversing the atomic wire defined by $V_{\text {eff }}$. For this purpose we have developed a transfer-matrix technique to solve the 3D quantum scattering problem based on a method reported in Ref. 25. From the scattering matrix we obtain conductance using the Landauer formula. ${ }^{26}$ As noted before, ${ }^{20,27}$ the crucial point of the two-step procedure is to include the long leads into the equilibrium analysis of the effective potential $V_{\text {eff }}$ which allows the eigenstates obtained from the ground-state density-functional calculation to be brought into the form of scattering states of the wire.

The details of the model and system parameters are as follows. ${ }^{28}$ The leads are modeled by the jellium model where the positive charges are uniformly distributed. A jellium lead has a cross section area of $7.25 \times 7.25$ (a.u. $)^{2}$, length $L$

$=30.78$ a.u., and its charge is specified ${ }^{29}$ by $r_{s} \approx 2.0$ a.u., mimicking metallic leads. This results in a total of 96 electrons in the jellium leads. The chain of $\mathrm{Si}$ atoms is sandwiched in between two leads. The atom-atom distance is fixed by the Si dimer bond length of 4.25 a.u. For the atomjellium distance, we used the value 2.3 a.u. which is the equilibrium bond length of a $\mathrm{Si}$ adatom on top of a jellium plane as determined in Ref. 29. The whole system (see inset of Fig. 2), atomic chain plus the leads, is put into a supercell of size $21.77 \times 21.77 \times(2 L+d)(\text { a.u. })^{3}$, which we checked to be large enough, for the standard plane-wave based $a b$ initio calculations $^{21}$ using a preconditioned conjugate gradient minimization scheme. Here $d$ is the length of the atomic chain including the atom-jellium distances. We have investigated atomic wires which contain up to eight $\mathrm{Si}$ atoms and used an upper energy cutoff of eight Ry.

Figure 1 shows the effective potential of a wire with six $\mathrm{Si}$ atoms. $V_{\text {eff }}$ in the 3D leads is affected by the atoms near the atom-lead junctions, but this effect is quickly screened out away from the junctions, indicating that the length of the leads is approaching the desired asymptotic limit. In the jellium leads, $V_{\text {eff }}$ is a potential well with a depth $\sim-0.5$ a.u. below the Fermi level in the cross section plane ( $x, y$ plane) for the six-atom wire. Similar values are obtained for other wires. In the atomic chain of the wire, Fig. 1 shows clear covalent bonding between the $\mathrm{Si}$ atoms. In addition, $V_{\text {eff }}$ has rather high peaks due to the ionic core of the atoms. Surrounding the cores there is the usual attractive part of atomic potential. Finally, the bonding between the Si atom and the jellium leads are also clearly obtained in Fig. 1, and it is quite similar to that of a $\mathrm{Si}$ adatom on top of a jellium plane. $^{29}$

Figures 2 and 3 show the conductance $G(E)$ as a function of the incoming electron energy $E$ for several wires with one to eight $\mathrm{Si}$ atoms in the chain. Several observations are in

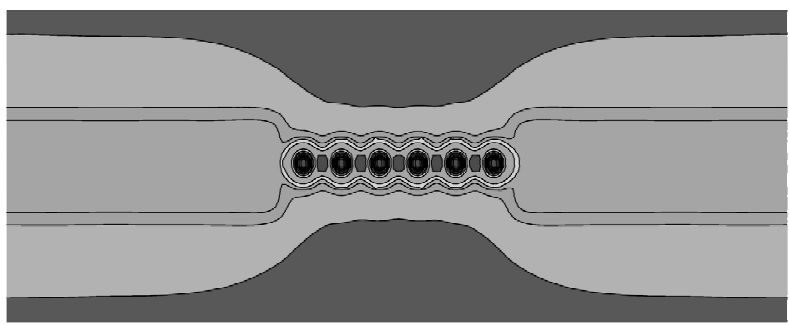

FIG. 1. The contour plot of the ground-state effective potential $V_{\text {eff }}$ obtained from the $a b$ initio total-energy calculation for a wire with six $\mathrm{Si}$ atoms. The covalent bonding between the $\mathrm{Si}$ atoms is clearly observed. The covalent bonding between a Si atom and the lead is less clear and is similar to that of an Si adatom on top of a jellium plane, reported in Ref. 29. Relative potential strength is shown by the lighter (darker) region which depicts lower (higher) values of $V_{\text {eff }}$. The scale ranges from $-14 \mathrm{eV}$ (white) to $+19 \mathrm{eV}$ (black), in intervals of $3.67 \mathrm{eV}$.

order. First, all wires show some degree of conductance quantization with clear conductance plateaus. ${ }^{30}$ However, the plateaus are not at perfect integer values of $2 e^{2} / h$ due to scattering at the atom-jellium junction which leads to finite reflection coefficients. This is similar to that of the experimental situation where parasitic series resistance in the leads reduces the total conductance. ${ }^{4}$ For $\mathrm{Si}$ wires the atom-jellium junction scattering is more severe as compared with that of Al wires which we studied previously. ${ }^{17}$ This may be understood since the $\mathrm{Si}$ atom closest to the leads makes a covalent bond with the next $\mathrm{Si}$ atom down the chain, while the covalent bond ${ }^{29}$ with a lead is certainly much weaker. Hence the charge density and $V_{e f f}$ is not symmetric on the two sides of this atom. Such an asymmetry in $V_{\text {eff }}$ leads to scattering of the incoming electron wave which produces a nonzero re-

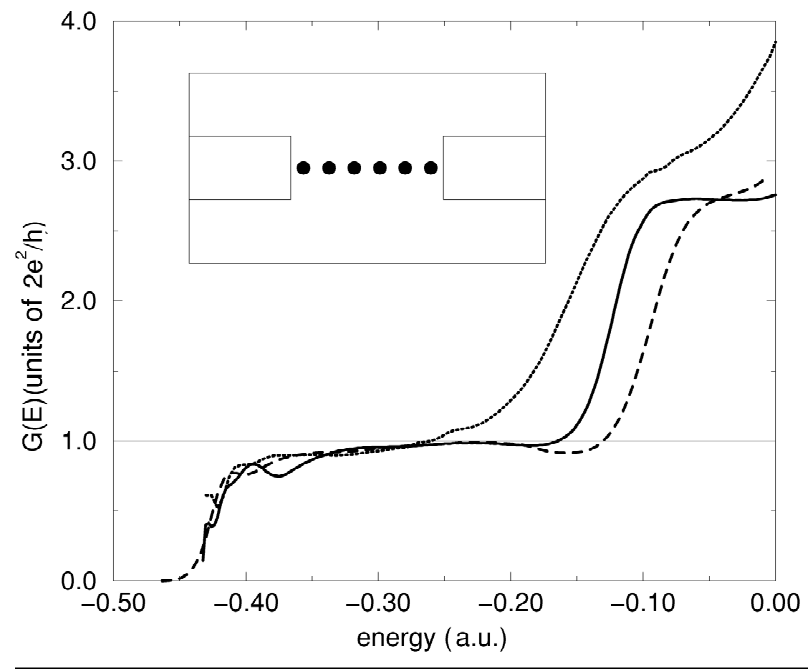

FIG. 2. Conductance $G(E)$ as a function of incoming electron energy $E$ for wires with one and two Si atoms. Dotted line: one $\mathrm{Si}$ atom wire. Solid line: two Si atom wire. Dashed line: two Si atom wire with larger cross section of the leads (50\% larger). The conductance shows two plateau regions. Inset: schematic plot of a long quantum wire which consists of a $\mathrm{Si}$ chain connected to two 3D jellium leads. The whole system is included in a supercell for planewave based $a b$ initio total-energy calculations. 


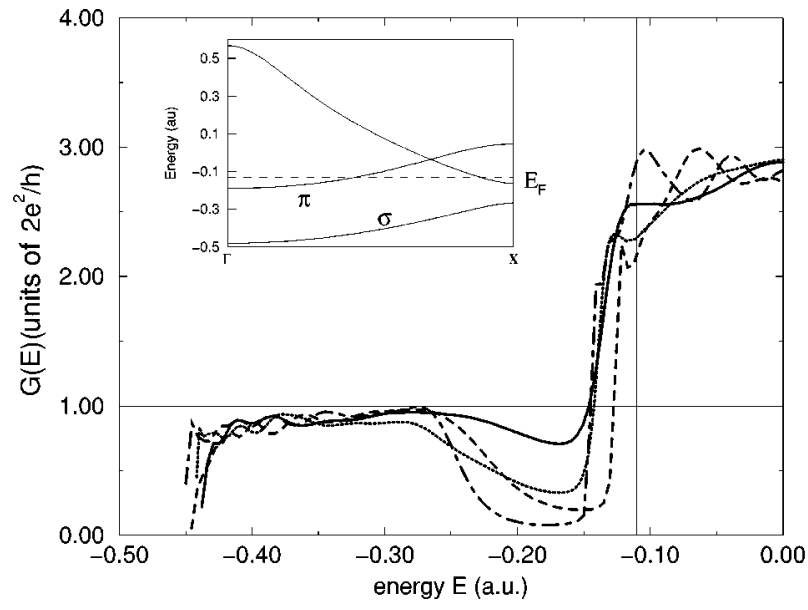

FIG. 3. Conductance $G(E)$ as a function of incoming electron energy $E$ for wires with more than two Si atoms. Solid: three atoms; dotted: four atoms; dashed: six atoms; dash-dotted: eight atoms. The vertical line indicates the position of the calculated Fermi level, $E_{f}$. Notice the development of the conductance dip below $E_{f}$ as the length of the atomic section increases. Inset: calculated 1D band structure of an infinitely long $\mathrm{Si}$ chain with bond length 4.09 a.u. The horizontal line is the calculated Fermi energy of the infinite chain. A gap exists below the Fermi level.

flection coefficient. The second feature of the conductance, as shown in Fig. 3, is the apparent dip of $G(E)$ near $E=$ -0.15 a.u. Such a dip is more pronounced for longer chains of $\mathrm{Si}$ atoms as if the wires were trying to develop a conductance gap. For short chains as shown in Fig. 2 such a dip has not developed. Our equilibrium density-functional analysis gives slightly different Fermi energies for different wires but the values are all near -0.11 a.u., well above this conductance dip as shown by the vertical line in Fig. 3. Thus the wires are certainly metallic. To understand where this conductance dip comes from, we have computed ${ }^{31}$ the 1D band structure of an infinitely long Si chain with bond length fixed at 4.09 a.u., as shown in the inset of Fig. 3. Indeed, the band structure shows a gap ${ }^{32}$ below the Fermi energy with a relative energy difference similar to that of Fig. 3. Hence the conductance dip observed here reflects the fact that an infinitely long Si chain has a band gap below the Fermi energy. The consistency between the general transport characteristics and the density of states (DOS) is actually expected since linear-response theory requires the conductance to be proportional to DOS. As a third feature we have investigated effects of the size of the jellium leads by increasing the cross section area of the leads. The dashed line of Fig. 2 is for a two-atom wire with the cross section area increased by $50 \%$. Comparing the solid and dashed curves of Fig. 2, for the wire with larger leads the onset of the second plateau is shifted to a higher energy. We have checked that the equilibrium Fermi energy of the wire with larger leads is also shifted upwards to $\sim-0.099$ a.u. However the main features of the conductance, namely the quantization, are the same for the two different sizes of the leads. Finally, the values of the atomic wire resistance can be obtained by inverting the conductance. At the Fermi level which is above but near the onset of the second plateau, the resistances for these wires have values ranging from the smallest $\sim 4.54 \mathrm{k} \Omega$ for the one atom case, to $\sim 7.28 \mathrm{k} \Omega$ for the wire with a wider lead.

To compare the results of $\mathrm{Si}$ system with those of $\mathrm{Al}$ system, ${ }^{17}$ while the main features of conductance quantization and the formation of quantum wires are similar, there exists several important differences. As discussed above, the $\mathrm{Si}$ atom-jellium junction provides scattering to the incoming electron which in turn produces nonzero reflection coefficients. This was due to a difference in the bonding between a $\mathrm{Si}$ atom to the leads and to its neighboring atom. For $\mathrm{Al}$ wires, the effective potential between an atom to the leads and to its neighboring atom is more spherical, thus the atomjunction scattering is not as severe, thereby producing more perfect conductance plateaus. ${ }^{17}$ Another difference is the conductance dip observed in Si wires; such a feature was not observed for $\mathrm{Al}$ wires consisting of up to four $\mathrm{Al}$ atoms in the atomic chain ${ }^{17}$ although such a conductance dip may still develop in $\mathrm{Al}$ wires with a longer atomic chain. Finally, the equilibrium Fermi energy for $\mathrm{Al}$ wires examined in Ref. 17 is located at the transition region between the first and second conductance plateau where $G(E)$ changes rapidly, leading to different resistances at the Fermi level for different wires. For Si wires studied here, the Fermi energy of the wires locates on the second conductance plateau, thus for all the wires examined the resistances at the Fermi energy have values within a smaller range.

To summarize, we have investigated the quantum transport properties of a model atomic wire made of a chain of $\mathrm{Si}$ atoms connected to two external long leads. Our prediction is based on a self-consistent $a b$ initio total-energy calculation of the effective ground-state potential of the wire, and a solution of a full 3D quantum scattering problem. Our results show that the Si atomic wire is metallic at its Fermi energy, and we have predicted the quantum conductance of the wires as a function of the incoming electron energy within the model. For all the wires studied some degree of conductance quantization is observed. A very interesting feature is the development of a conductance dip below the Fermi energy of the atomic wire as the length of the atomic section is increased. This behavior can be explained by the existence of a gap in the infinitely long $1 \mathrm{D} \mathrm{Si}$ chain band structure. Finally we comment that the curves of $G=G(E)$ are useful for giving a first estimate of the electrical current as a function of a voltage across the wire: one obtains the current in the usual fashion by integrating $G(E)$ over energy convoluted with a Fermi function. As mentioned in the Introduction, Si based freestanding atomic wires with sizes in the nanometer range have been fabricated using several experimental techniques. On the other hand our model studied here is for ideal chains of $\mathrm{Si}$ atoms connected to external leads. Such chains may be produced using the atomic manipulation ability of a STM on top of a substrate. Clearly, a transport measurement of such a system will be extremely useful and interesting.

We gratefully acknowledge financial support by NSERC of Canada and FCAR of Quebec. J. Wang was supported by a RGC grant of the Hong Kong Government under Grant No. HKU 261/95P, and a research grant from the Croucher Foundation. We thank the Computing Center of University of Hong Kong for a substantial CPU allocation. 
*Present address: Fritz-Haber-Institut der Max-PlanckGesellschaft, Farada yweg 4-6, D-14195 Berlin-Dahlem, Germany.

${ }^{1}$ L. T. Canham, Appl. Phys. Lett. 57, 1046 (1990).

${ }^{2}$ Hideo Namatsu, Yasuo Takahashi, Masao Nagase, and Katsumi Murase, J. Vac. Sci. Technol. B 13, 2166 (1995).

${ }^{3}$ Yasuo Wada et al., J. Vac. Sci. Technol. B 12, 48 (1994).

${ }^{4}$ Y. Nakajima et al., Appl. Phys. Lett. 65, 2833 (1994).

${ }^{5}$ Masanobu Miyao, Jpn. J. Appl. Phys., Part 1 33, 7214 (1994).

${ }^{6}$ Yasuo Wada et al., J. Appl. Phys. 74, 7321 (1993).

${ }^{7}$ J. I. Pascual et al., Science 267, 1793 (1995); J. Vac. Sci. Technol. B 13, 1280 (1995); Phys. Rev. Lett. 71, 1852 (1993).

${ }^{8}$ L. Kuipers and J. W. M. Frenken, Phys. Rev. Lett. 70, 3907 (1993)

${ }^{9}$ E. S. Snow, D. Park, and P. M. Campbell, Appl. Phys. Lett. 69, 269 (1996).

${ }^{10}$ M. Brandbyge et al., Phys. Rev. B 52, 8499 (1995).

${ }^{11}$ Ali Yazdani, D. M. Eigler, and N. D. Lang, Science 272, 1921 (1996).

${ }^{12}$ T. N. Todorov and A. P. Sutton, Phys. Rev. Lett. 70, 2138 (1993).

${ }^{13}$ A. Garcia-Martin, J. A. Torres, and J. J. Sáenz, Phys. Rev. Lett. 77, 2245 (1996); Phys. Rev. B 54, 13448 (1996).

${ }^{14}$ N. D. Lang, Phys. Rev. B 52, 5335 (1995).

${ }^{15}$ A. J. Read, et al., Physica A 207, 411 (1994); A. J. Read et al., Phys. Rev. Lett. 69, 1232 (1992).

${ }^{16}$ F. Buda, J. Kohanoff, and M. Parrinello, Phys. Rev. Lett. 69, 1272 (1992).

${ }^{17}$ C. C. Wan, J.-L. Mozos, Jian Wang, and Hong Guo, Appl. Phys. Lett. 71, 419 (1997); the ac properties of Al atomic wires is in Phys. Rev. B 55, R13 393 (1997).

${ }^{18}$ H. Mehrez, S. Ciraci, A. Buldum, and Inder P. Batra, Phys. Rev. B 55, R1981 (1997).
${ }^{19}$ S. Watanabe, Y. A. Ono, T. Hashizume, Y. Wada, J. Yamanchi, and M. Tsuhada, Phys. Rev. B 52, 10768 (1995).

${ }^{20}$ Yongjiang Wang, Jian Wang, Hong Guo, and E. Zaremba, Phys. Rev. B 52, 2738 (1995).

${ }^{21}$ M. C. Payne, M. P. Teter, D. C. Allan, T. A. Arias, and J. D. Joannopoulos, Rev. Mod. Phys. 64, 1045 (1992); G. P. Srivastava and D. Weaire, Adv. Phys. 36, 463 (1987).

${ }^{22}$ N. Govind, José-Luis Mozos, and Hong Guo, Phys. Rev. B 51, 7101 (1995); N. Govind, Jian Wang, and Hong Guo, ibid. 50, 11175 (1994).

${ }^{23}$ S. Goedecker, M. Teter, and J. Hutter, Phys. Rev. B 54, 1703 (1996).

${ }^{24}$ J. Ihm and M. L. Cohen, Solid State Commun. 29, 711 (1979).

${ }^{25}$ W. D. Sheng and J. B. Xia, Phys. Lett. A 220, 268 (1996).

${ }^{26}$ R. Landauer, IBM J. Res. Dev. 1, 233 (1957); Z. Phys. B 68, 217 (1987).

${ }^{27}$ M. Büttiker, in Quantum Dynamics of Submicron Structures, edited by H. A. Cerderia (Kluwer, Netherlands, 1995), p. 657.

${ }^{28} \mathrm{We}$ present results in atomic units throughout this work.

${ }^{29}$ N. D. Lang and A. R. Williams, Phys. Rev. B 18, 616 (1978).

${ }^{30}$ Notice that the plateau at $2 \sim 2 e^{2} / h$ is missing, which is a result for $3 \mathrm{D}$ wires. For a perfect $3 \mathrm{D}$ wire with a square cross section, the transverse quantization gives conductance steps of $1,3,4,6$, $\ldots$ in units of $2 e^{2} / h$.

${ }^{31}$ We have used the $a b$ initio method of Sankey and Niklewski to compute the 1D band structure. See O. F. Sankey and D. J. Niklewski, Phys. Rev. B 40, 3979 (1989).

${ }^{32}$ The band structure of an infinitely long 1D Si chain has been computed before using a tight-binding theory. See, for example, Toshishige Yamada and Yoshihisa Yamamoto, Phys. Rev. B 54, 1902 (1996). Our results, shown in the inset of Fig. 3, are consistent with theirs. 